\title{
Unsupervised Segmentation of Breast Infrared Images in Lateral View Using Histogram of Oriented Gradients
}

\author{
Thays Lacerda Correa*, Fabíola Freitas de Oliveira *, Matheus de Freitas Oliveira Baffa ${ }^{\dagger}$, Lucas Grassano Lattari * \\ *Instituto Federal de Educação, Ciência e Tecnologia do Sudeste de Minas Gerais (IF Sudeste MG) \\ Rio Pomba, MG, Brasil \\ †Universidade de São Paulo (USP) \\ Ribeirão Preto, Brasil \\ Email: thays.lacerdac@gmail.com, ffabiolafreitas@gmail.com,mfreitas826@gmail.com, lucas.lattari@ifsudestemg.edu.br
}

\begin{abstract}
Resumo-Breast cancer is the second most common type of cancer in the world. It is estimated that $29.7 \%$ of new cases diagnosed in Brazil occur in any structures of the breasts. However, the disease has a good prognosis if detected early. Thus, the development of new technologies to help doctors to provide an accurate diagnosis is indispensable. The goal of this work is to develop a new method to automate parts of computer-aided diagnosis systems, performing the unsupervised segmentation of the Region of Interest (ROI) of infrared breast images acquired in lateral view. The segmentation proposed in this paper consists of three stages. The first stage pre-processes the infrared images of the lateral region of breasts. Later, features are extracted from a descriptor based on Histogram of Oriented Gradients (HOG). Concluding, a Machine Learning algorithm is used to perform the segmentation of the sample. The current method obtained an average of $89.9 \%$ accuracy and $94.3 \%$ specificity in our experiments, which is promising compared to other works.

Index Terms-breast cancer, histogram of oriented gradients, image segmentation, infrared imaging, computer vision, computer aided diagnosis
\end{abstract}

\section{INTRODUÇÃO}

O carcinoma mamário, ou câncer de mama, é uma doença caracterizada pelo surgimento de uma neoplasia em algumas das estruturas que compõem a mama. Esta pode atingir homens e mulheres, geralmente acima dos 35 anos, sendo mais frequentes em mulheres após o período da menopausa [9].

De acordo com o Instituto Nacional de Câncer (INCA), o câncer de mama é o segundo tipo mais frequente [11]. Estimase que $29,7 \%$ dos novos casos de câncer registrados no Brasil, dentre as mais de 100 variações, sejam do tipo mamário e, embora raro, $1 \%$ do total de casos ocorrem em homens. Além disto, estima-se que uma em cada oito mulheres desenvolverão a doença em algum momento de sua vida [7], [8].

Desde 2013 notou-se um aumento de 4,7\% na taxa de incidência da doença no Brasil. Embora o número de casos registrados tenha aumentado, o número de mortes pelo câncer têm reduzido. Este comportamento é visível quando se compara a taxa de incidência do câncer de mama e sua taxa de mortalidade, por ano, entre os países desenvolvidos e em desenvolvimento [3]. Isso ocorre devido à detecção precoce da doença, possibilitando tratamentos menos agressivos, como os tratamentos sistêmicos e proporcionando uma melhor qualidade de vida ao paciente.

Para o ano de 2018, o INCA registrou, aproximadamente, 17 mil mortes pelo câncer de mama no Brasil. Esse valor é referente a aproximadamente $16,4 \%$ dos casos registrados [7]. Embora possua uma alta taxa de incidência e óbito, o câncer de mama também é considerado o tipo com melhor prognóstico, possibilitando ao paciente até $95 \%$ de chances de cura quando diagnosticado precocemente [6], [7].

Existem diferentes maneiras de se diagnosticar o câncer de mama. Um desses meios é através do autoexame, no qual a própria pessoa percebe sinais de anomalias ou a presença de caroços na pele. Outra forma de detecção é através dos exames por imagem, que possuem um alto grau de eficácia. Um exemplo de exame por imagem bastante utilizado é a mamografia, que emprega Raios-X para registrar as estruturas internas da mama e, assim, localizar possíveis anormalidades.

As imagens de infravermelho ou termografia, por sua vez, possuem grande potencial para a detecção precoce dessa doença. Isso ocorre devido ao processo de formação deste tipo de neoplasia. Diversos fatores como a atividade metabólica e o processo de angiogênese fazem com que determinadas regiões da mama produzam calor de forma anormal e contribuem com desbalanceamento da distribuição térmica entre as mamas [1], [10]. Além disto, as imagens de infravermelho podem ser sintetizadas utilizando dispositivos mais baratos economicamente e menos desconfortáveis quando comparados por exemplo, com o mamógrafo e a máquina de Ressonância Magnética, equipamentos amplamente usados em exames de imagem.

Neste âmbito, sistemas de apoio ao diagnóstico (do inglês, Computer-Aided Diagnosis - CAD) são desenvolvidos com o intuito de prover um diagnóstico mais preciso e eficaz. Considerando a disponibilidade de dados e o poder de processamento das máquinas atuais, é viável o desenvolvimento de sistemas CAD que integram imagens infravermelhas e algoritmos de Aprendizado de Máquina sofisticados. 
Típicos sistemas CAD são compostos por quatro etapas, sendo elas (i) a Pré-Processamento de Imagens, (ii) a Segmentação da Região de Interesse (ROI - Region of Interest), (iii) a Extração de Atributos ou Características (features) e (iv) a Classificação [12]. No primeiro estágio pode-se aplicar algum procedimento que realce ou reduza alguma característica da imagem originalmente obtida, a fim de se potencializar os resultados das etapas posteriores. A seguir, a etapa de Segmentação remove toda a informação não necessária para a análise da imagem a posteriori, como o fundo, permanecendo apenas a ROI. Na terceira etapa, informações relevantes para a análise são extraídas. Por fim, as características extraídas são usadas para o treinamento de algoritmos de Aprendizado de Máquina que produzirão modelos de classificação para que se possa diagnosticar exames, considerando um conjunto de amostras de pacientes saudáveis e doentes.

Esse trabalho propõe uma metodologia para a etapa de Segmentação da ROI de Imagens Infravermelhas das Mamas atendendo ao protocolo lateral, em que o registro de fotos é rotacionado em $90^{\circ}$. Essa proposta é não supervisionada (automática) e sua avaliação metodológica é feita com imagens advinda de bases de dados médicas públicas. Para ilustrar, a Figura 1 demonstra exemplos de imagens dos protocolos mais comumente adotados, que são: o frontal e o lateral. A Figura 2 ilustra qual o tipo de imagem de entrada utilizada e sua saída esperada (segmentação da ROI).
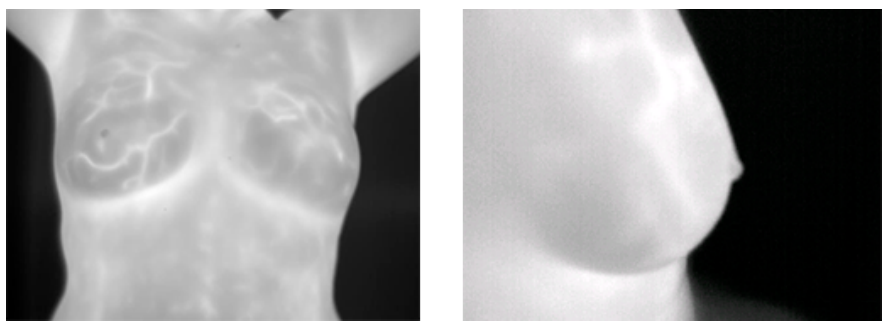

Figura 1: Imagens infravermelhas das mamas adquiridas com o protocolo frontal (à esquerda) e lateral (à direita). Fonte: autor.
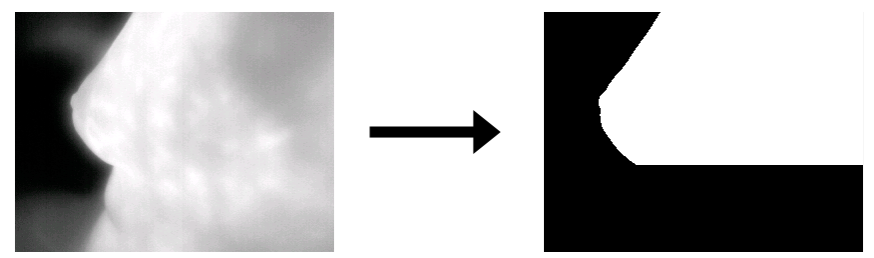

Figura 2: Exemplo de imagem infravermelha usada (à esquerda) e segmentação da ROI esperada que o método realize (à direita). Fonte: autor.

Algumas contribuições dessa proposta:

- Descrição de um novo método para a segmentação automática de imagens em protocolo lateral das mamas. Isso é importante considerando que um número diminuto de pesquisas envolvendo o protocolo lateral são desenvolvidas.

- Possibilidade de generalização da proposta para problemas com outras regiões de interesse.

- Discussão e comparação qualitativa com outras estratégias que empregam o protocolo lateral.

- Apresentação de resultados numéricos competitivos com outros trabalhos que lidam com o mesmo problema.

- Portabilidade para outras arquiteturas dada a utilização de algoritmos amplamente conhecidos e adotados, como é o caso de Histograma de Gradientes Orientados [4].

Este trabalho está organizado da seguinte forma: a Seção II retrata o estado da arte em segmentação de imagens infravermelhas laterais das mamas; as seções III e IV, respectivamente, detalham o método proposto, os experimentos empregados, o desempenho quantitativo dos mesmos e suas limitações; finalmente, a Seção V apresenta as conclusões obtidas e os trabalhos futuros da proposta.

\section{TRABAlhos Relacionados}

A suma maioria das metodologias de segmentação de imagens infravermelhas são desenvolvidas com maior enfoque para imagens frontais das mamas [2], [16], [18]. No entanto, o escopo deste trabalho objetiva produzir resultados em imagens sob uma visualização em ângulos distintos da região das mamas (protocolo lateral). É de comum conhecimento que a visualização de imagens médicas é sensível à posição que o exame é gerado ou ao recorte realizado, trazendo diferentes informações conforme o ângulo se altera.

Em Oliveira [19] é proposta uma metodologia de segmentação automática de imagens infravermelhas laterais. Inicialmente, as 328 imagens utilizadas pelo autor são convertidas em preto e branco (pré-processamento). Em seguida, são identificados e eliminados os elementos indesejáveis no fundo das imagens. Por fim, a última etapa é responsável pela detecção e refinamento de cantos, identificando a prega inframamária e, assim, atingindo a segmentação das imagens automaticamente. O método obteve uma acurácia de $93 \%$ e especificidade de $96 \%$.

Recentemente, o trabalho de Josephine et al. [13] propõe um fluxo de trabalho que consiste em: remover informações textuais e de temperatura por meio de um algoritmo in-paint, realce de imagens por meio de um filtro de difusão anisotrópico e extração de ROI por meio de um algoritmo baseado na abordagem de level sets. Para avaliação de resultados, foram usadas as métricas de índice de Jaccard, coeficiente de Sørensen-Dice, índice de Similaridade Estrutural (Structural Similarity Index), dentre outros. Apenas 15 imagens foram usadas em sua proposta, obtendo $95 \%$ de correlação entre as áreas segmentadas pelo trabalho e as regiões reais esperadas.

Outro trabalho de suma importância fora apresentado por Morales-Cervantes et al [17], que combinaram imagens de protocolos laterais e frontais em seu método. Por meio de uma abordagem que emprega o filtro Sobel e o espaço de cor $\mathrm{L} * \mathrm{a} * \mathrm{~b} *$, estes obtiveram uma sensibilidade de $100 \%$ e acurácia de $69,9 \%$. 
Finalmente, pode-se mencionar os trabalhos de Santana et al. [5], Mambou et al. [15] e Kakileti et al. [14] que, ainda que não estejam diretamente relacionados com a segmentação de imagens das mamas usando protocolo lateral, são propostas recentes importantes para a literatura. Respectivamente, o primeiro é um estudo profundo sobre classificação de imagens de infravermelho (inclusive com o protocolo lateral), o segundo aplica a classificação diagnóstica utilizando Aprendizado Profundo (Deep Learning) e o último descreve os avanços recentes envolvendo diagnóstico de imagens infravermelhas das mamas em formato survey.

\section{Materiais e Métodos}

Essa seção será subdividida em três partes. A primeira subseção descreve a base de dados empregada para os experimentos a fim de validar a proposta; a segunda subseção enfatiza o funcionamento do classificador utilizado para reconhecer determinadas seções da imagem; finalmente, a última parte explica como funciona o estágio de segmentação de imagens do trabalho proposto.

\section{A. Base de Dados}

$\mathrm{O}$ método proposto neste trabalho foi testado em um banco público de imagens da UFF/UFPE que encontra-se disponível em PROENG [20]. Estas imagens são proveniente de estudos realizados por pesquisadores da Universidade Federal de Pernambuco e seus parceiros. Essa base de dados contém imagens com protocolo estático e foram capturadas pelo Hospital das Clínicas da mesma. Na base supracitada, há imagens infravermelhas capturadas em oito ângulos distintos. No entanto, no contexto desse trabalho, apenas as imagens de infravermelho das laterais das mamas foram empregadas no processo de segmentação, que possuem ângulo de $90^{\circ}$. Devido a algumas inconsistências na base, como falta de informações sobre o diagnóstico das pacientes, algumas imagens foram eliminadas dessa análise. No total, 214 imagens foram utilizadas para os experimentos apresentados nas seções III-B e III-C.

Inicialmente, são usadas imagens limiarizadas da base original, convertidas para tons de cinza. Esse processo produz uma imagem em formato bitmap.

\section{B. Classificação do Modelo e Reconhecimento de Contorno das Pregas Inframamárias}

Após obter as imagens de infravermelho em preto e branco, segmentos das mesmas são extraídos manualmente das imagens originais. Isso faz-se necessário para assim treinar um modelo classificador que distinguirá regiões diversas das imagens das mamas.

Uma região fundamental para o funcionamento desse métodos são os cantos contendo pregas inframamárias. Um exemplo de tal região é assinalada na Figura 3.

Conjuntos de segmentos extraídos manualmente são então introduzidos em dois subconjuntos: imagens cujo canto da prega inframamária encontra-se na posição central da imagem e imagens com segmentos diversos das mamas que não contenham cantos. Chamaremos esses conjuntos, respectivamente,

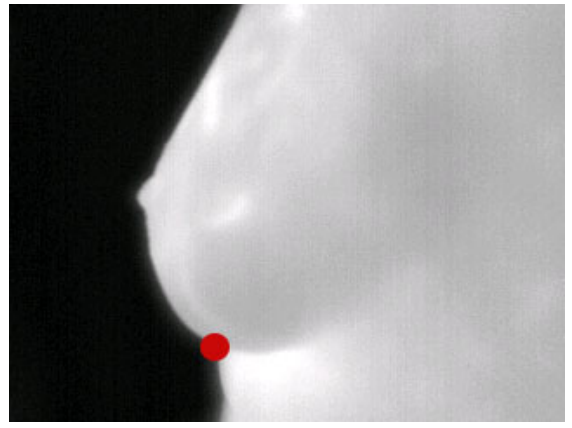

Figura 3: Amostra com as regiões de canto consideradas na análise descrita nessa seção. Fonte: autor.

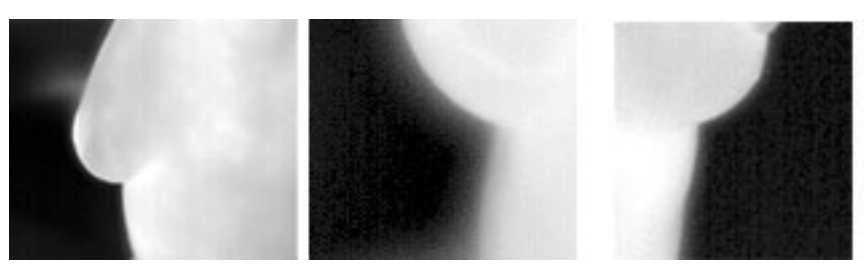

(a) Imagens introduzidas em $I M G S_{\text {pos }}$.
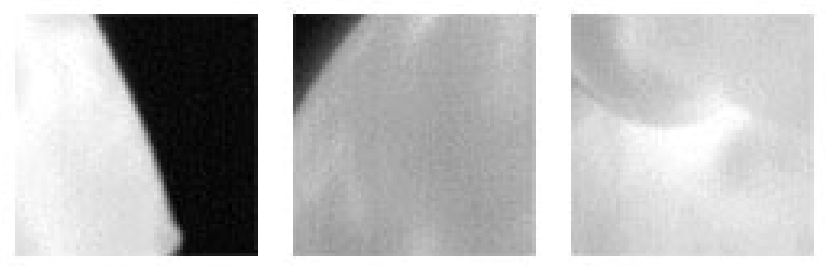

(b) Imagens introduzidas em $I M G S_{n e g}$.

Figura 4: Exemplos de imagens usadas para treinar o modelo classificador dessa seção. Em (a), o canto das imagens (prega inframamária) estão centralizados, a fim de se especificar ao modelo que essas regiões precisam ser localizadas nas amostras de entrada. Quanto a (b), tem-se imagens sem os cantos assinalados na região central. Dessa forma, o modelo irá compreender que não se deve assinalar que essas sejam regiões rotuladas como cantos. Fonte: autor.

de $I M G S_{p o s}$ e $I M G S_{n e g}$ (Figura 4). Nessa proposta, 61 segmentos fazem parte de $I M G S_{\text {pos }}$ e 189 segmentos foram atribuídos a $I M G S_{n e g}$.

Os segmentos contidos em $I M G S_{\text {pos }}$ e $I M G S_{n e g}$ serão parte do conjunto de treino de um classificador descrito por Histogramas de Gradientes Orientados. Esse descritor foi originalmente proposto para o reconhecimento automático de pedestres em imagens [4]. Esse vetor de características é bastante utilizado em problemas de Visão Computacional para detectar ou reconhecer objetos dos mais diversos que se diferenciem visualmente, principalmente por meio da geometria e textura. Sua proposta baseia-se em um conjunto robusto de características para discriminar regiões com variações de iluminação e pose.

Para o treinamento do classificador, regiões diversas das 
imagens são recortadas atendendo a um tamanho padrão. Esses recortes possuem aproximadamente $30 \%$ da área original dessas imagens, sendo distinguidas manualmente em $I M G S_{\text {pos }}$ e $I M G S_{n e g}$.

A seguir, são computados os descritores de Histograma de Gradientes Orientados de cada uma das imagens de ambos os conjuntos. Os parâmetros aplicados são: 8 histogramas de orientação dos gradientes, grades de células da ordem de $16 \times 16$ pixels, $4 \times 4$ células por bloco e, finalmente, os blocos são normalizados usando a norma L2. Essa configuração é tradicional para problemas desse tipo e funciona adequadamente para a atual proposta, conforme apresentado na seção de resultados (Seção IV).

Estes descritores das imagens são vetorizados e introduzidos como amostras em um classificador baseado em Máquina de Vetor Suporte (Support Vector Machine - SVM). O SVM corresponde a um algoritmo de Aprendizado de Máquina supervisionado que pode ser usado tanto para classificação quanto para regressão. Nesse artigo, o SVM é empregado para fins de classificação, com os seguintes parâmetros: o termo de penalidade $C$ (usado para ajustar a distância entre seu hiperplano separador e a primeira amostra de cada classe $I M G S_{p o s}$ e $I M G S_{n e g}$ ) é da ordem de 1.0. A função de kernel empregada é a RBF e o coeficiente de kernel $\gamma$ é computado automaticamente (de forma que o mesmo é $\frac{1}{n_{f}}$, em que $n_{f}$ é o número de características advindas dos descritores). A importância de $\gamma$ define a influência em que uma única amostra do treinamento terá no modelo como um todo. Após aplicado o SVM, tem-se o modelo $M$.

Finalmente, $M$ será usado para reconhecer automaticamente o segmento $s_{i}$ que contém os candidatos a canto da prega inframamária de cada uma das imagens $i$. Espera-se que o canto esteja centralizado em $s_{i}$. Uma vez encontrado, anota-se uma coordenada local $(x, y)$ exatamente na posição em que se encontra o canto em $s_{i}$ para que seja usada na etapa seguinte, de segmentação. Assim, ao final da etapa de classificação, temse as posições $x$ e $y$ que indicam a localização de cada prega inframamária de cada amostra $i$. Alguns exemplos dessas imagens encontram-se apresentadas na Figura 5.

\section{Segmentação das Imagens de Infravermelho}

$\mathrm{Na}$ etapa de segmentação cada imagem da base de dados é analisada e, de cada uma, é extraída a região lateral contendo a mama. Alguns exemplos de imagens segmentadas pelo método podem ser vistas na Figura 6.

Inicialmente, computa-se a binarização da imagem original em formato de tons de cinza. O limiar empregado que comportou-se bem na proposta foi o de 150, considerando que inicialmente cada imagem possui 8 bits de nível de cor (255).

A seguir, computa-se o histograma de linha $h_{s i}(y)$ da versão computada $s_{i}$ no passo anterior (Seção III-B). Como é dito, cada $s_{i}$ contém a região da prega inframamária centralizada, tendo sido detectada por meio do modelo SVM usando o descritor HOG. Devido ao fato de, usualmente, a região da prega inframamária conter a menor densidade do histograma
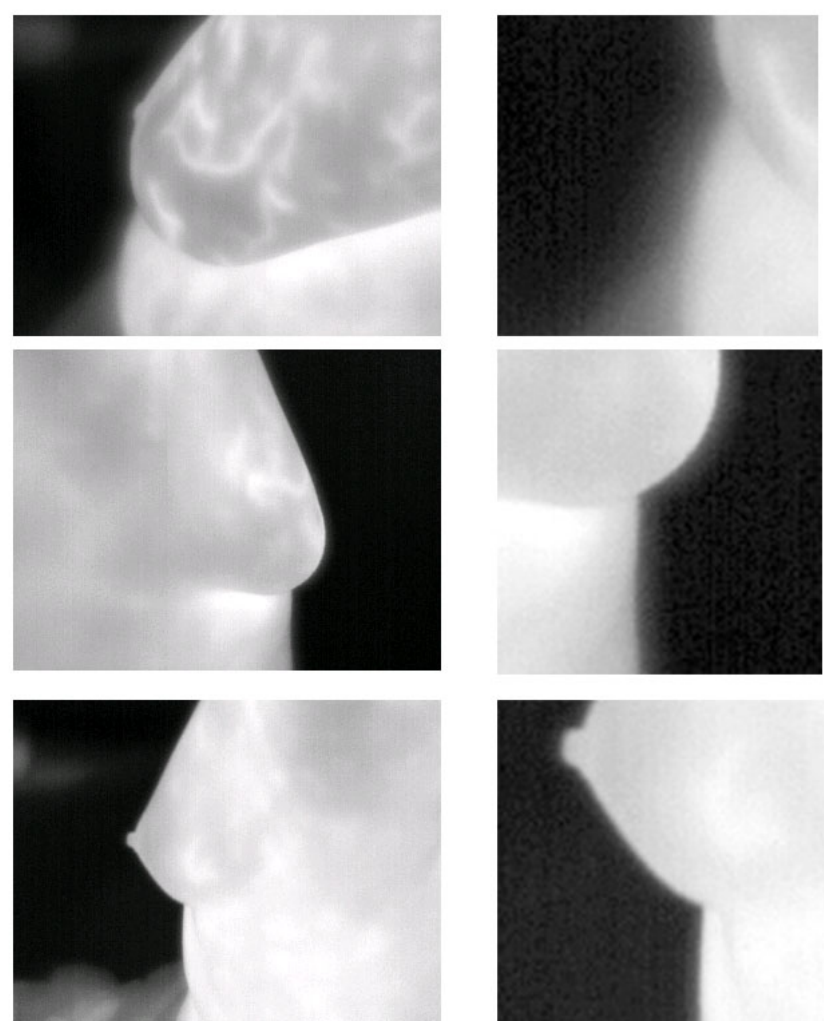

Figura 5: Algumas imagens da base de dados de mama lateral e suas versões computadas contendo a região da prega inframamária próxima a sua região central. Fonte: autor.

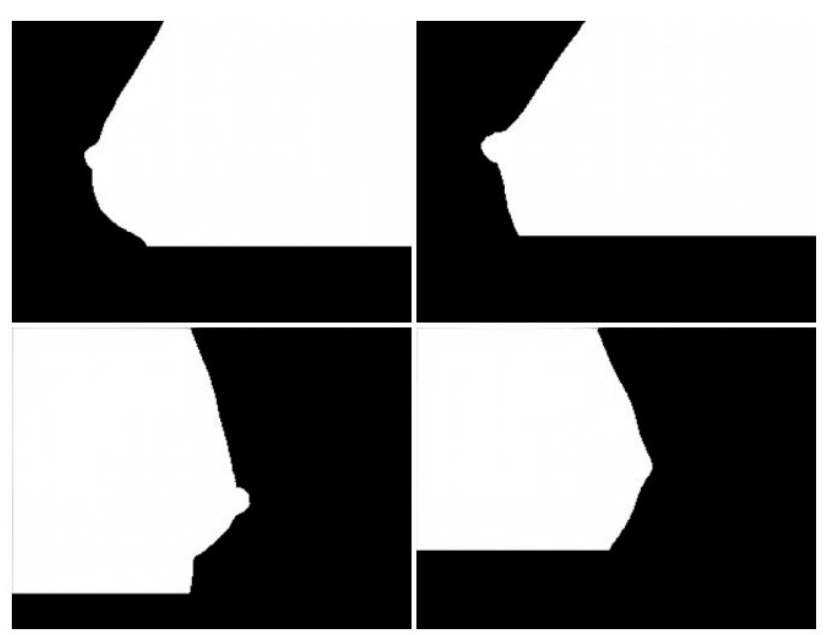

Figura 6: Exemplo de amostras segmentadas pelo método proposto. Fonte: autor.

de linha, este é um bom indicativo para localizar essa região. Assim, a coordenada $y$ contendo o $h_{s i}(y)$ de valor mínimo para $s_{i}$ sinaliza a posição vertical em que se localiza a prega inframamária.

No entanto, convém mencionar que deve-se iniciar a computação de $h_{s i}(y)$ na posição $y=0.3 \times i_{\text {altura }}$ da imagem, sendo que $i_{\text {altura }}$ é a altura de $i$. Isso é necessário pois, anali- 
sando empiricamente, foi constatado que, na figura mamária, a prega inframamária encontra-se costumeiramente na região limítrofe inferior da imagem. Todos os pixels localizados em posições iguais ou abaixo de $y$ na imagem original $i$ em que foi detectada a prega inframamária são sumariamente removidos da imagem. As constantes apresentadas aqui foram calculadas empiricamente,, após exaustivos experimentos a fim de obter o melhor resultado. Um exemplo de tal procedimento é demonstrado na Figura 7.
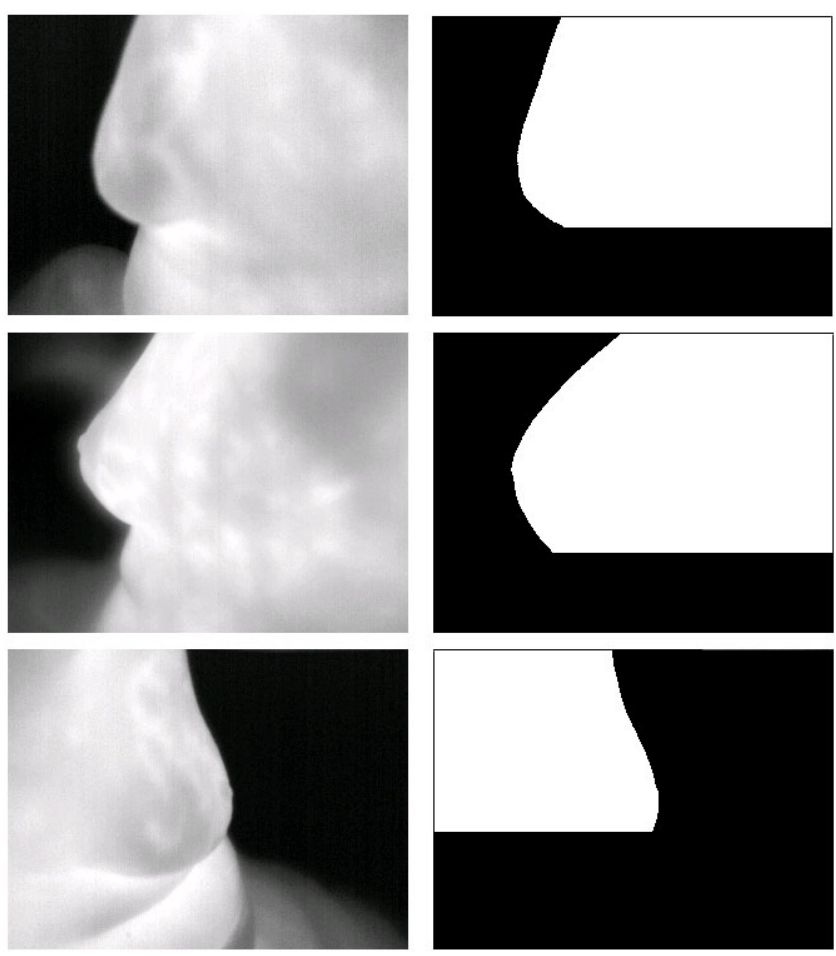

Figura 7: Comparativo entre a imagem original e sua contraparte após a remoção dos pixels proporcionada pela etapa de segmentação. Fonte: autor.

\section{Resultados E Discussões}

A avaliação do método proposto baseia-se na comparação dos resultados numéricos obtidos com as segmentações automáticas realizadas. Uma análise quantitativa foi realizada utilizando as seguintes métricas de avaliação quantitativa: acurácia, sensibilidade, especificidade, preditividade positiva e preditividade negativa.

A Tabela I contém um comparativo dos resultados obtidos neste trabalho em contraste com os resultados obtidos por Oliveira [19] para a mesma base de imagens infravermelhas das laterais da mama utilizadas neste trabalho. Ambos os trabalhos compararam as segmentações automáticas resultantes utilizando uma segmentação manual previamente realizada por um médico especialista para fins de comparação.

Considerou-se também os resultados obtidos por MoralesCervantes et al. [17], ainda que não tenham sido usadas as mesmas imagens em nossos experimentos, visto que as
Tabela I: Comparação de resultados entre o trabalho de Oliveira [19] e o método proposto usando a mesma base de dados. Fonte: autor.

\begin{tabular}{|c|c|c|}
\hline Métrica & Oliveira [19] & Este Trabalho \\
\hline Acurácia & $93 \%$ & $89,6 \%$ \\
\hline Sensibilidade & $95 \%$ & $85,7 \%$ \\
\hline Especificidade & $96 \%$ & $94,3 \%$ \\
\hline Preditividade Positiva & $96 \%$ & $92,7 \%$ \\
\hline Preditividade Negativa & $96 \%$ & $89,1 \%$ \\
\hline
\end{tabular}

Tabela II: Comparação de resultados entre o trabalho de Morales et al. [17] e o método proposto sem que seja usada a mesma base de dados. Fonte: autor.

\begin{tabular}{|c|c|c|}
\hline Métrica & Morales-Cervantes [17] & Este Trabalho \\
\hline Acurácia & $69,9 \%$ & $89,6 \%$ \\
\hline Sensibilidade & $100 \%$ & $85,7 \%$ \\
\hline Especificidade & Não avaliado & $94,3 \%$ \\
\hline Preditividade Positiva & $11,42 \%$ & $92,7 \%$ \\
\hline Preditividade Negativa & $100 \%$ & $89,1 \%$ \\
\hline
\end{tabular}

imagens usadas por eles não encontram-se publicamente disponíveis. A comparação do método proposto com o trabalho de Morales-Cervantes et al. pode ser visto na Tabela II. Esse problema também ocorreu em relação ao trabalho de Josephine et al. [13], com o agravante das métricas adotadas serem distintas das nossas. De qualquer forma, optou-se por comparálas para mostrar resultados de outras aplicações envolvendo segmentação de imagens médicas das mamas usando protocolo lateral.

O método proposto nesse artigo (Seção III) foi implementado usando a linguagem Python em sua versão 3.5.4 x64 com as bibliotecas OpenCV para manipulação de imagens e Scikit-Learn para a utilização do Histograma de Gradientes Orientados e da Máquina de Vetor Suporte. O equipamento empregado para a aquisição de resultados foi um computador com processador Intel Core i5-2450M com 2.50Ghz e 6GB de Memória RAM, com sistema operacional Windows 7 Home Premium.

Nessa proposta, a acurácia da segmentação aproximou-se de $90 \%$, sendo bastante competitiva com relação ao trabalho de Oliveira [19]. A sensibilidade, por sua vez, apresenta uma relação para a classificação correta dos pixels considerados positivos nas imagens. Dessa forma, essa métrica mostra o quanto de acerto houve com relação aos pixels que são efetivamente pertencentes as mamas. A porcentagem de $85,7 \%$ descreve que o método proposto acarreta em maiores erros ao classificar os pixels que pertencem às regiões mamárias do que o outro trabalho comparado.

Um caso típico de falha do método refletido na sensibilidade é visível na Figura 8(a). Ao aplicar a limiarização na figura 8(a), tem-se a imagem 8(b). Nela, é possível ver que a posição y com o histograma de linha com valor mínimo está fora da prega inframamária (Figura 8(c)). O resultado esperado encontra-se na imagem 8(d).

Por sua vez, a proporção de acertos considerando apenas os pixels que pertencem aos verdadeiros negativos (fundo da imagem e região abaixo da prega inframamária) apresentam bom 


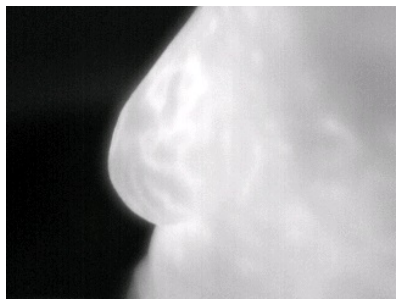

(a)

(c)

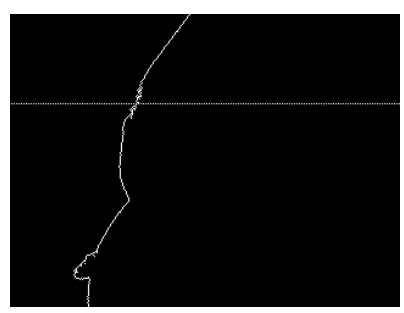

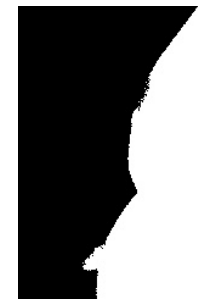

(b)

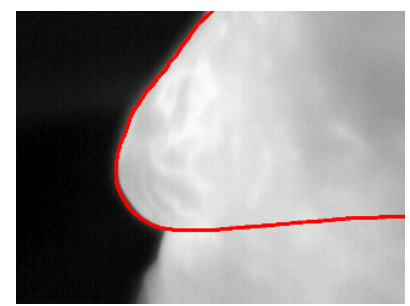

(d)
Figura 8: Avaliação visual do método considerando uma típica limitação. Em (a) a imagem original (IR_0832_grey.jpg). Em (b) a mesma imagem após a limiarização com limiar de 150. A imagem (c) contém a linha em destaque que demarca a posição $y$ em que o método incorretamente traçou a prega inframamária. E em (d), a marcação manual demonstrando qual seria a segmentação correta. Fonte: autor.

desempenho. Isso é demonstrado na métrica de especificidade, que apresentou o valor de $94,3 \%$.

Finalmente, as medidas de preditividade possuem taxas próximas a $90 \%$, o que possibilita ao método a predição potencialmente correta dos pixels pertencentes ou não a região mamária.

\section{CONClusÕes}

Nesse trabalho foi apresentada uma proposta para realizar segmentação automática de imagens infravermelhas das mamas utilizando o protocolo lateral. Este se baseia em ferramentas simples bastante conhecidas da Visão Computacional, como é o caso da Máquina de Vetores Suporte e Histograma de Gradientes Orientados. Esse processo será de suma importância para análise de imagens infravermelhas das mamas e para auxiliar em diagnósticos médicos de enfermidades nas mamas, como é o caso do câncer.

A partir dos resultados obtidos em experimentos, o resultado final aproximou-se de $90 \%$, o que possibilita um desempenho razoável para automatizar o processo de separação entre região objeto e fundo (mama e não mama). Entretanto, abre-se uma margem razoável de melhoria, sendo essa uma das expectativas com relação ao andamento desse projeto.

Os projetos futuros, por sua vez, podem ser descritos da seguinte maneira, (i) utilizar outro meio de se assinalar a prega inframamária, ao invés de usar histograma de linha. Uma possibilidade é avaliar a eficácia do SIFT (Scale-invariant feature transform), (ii) considerar o uso de propostas de segmentação baseadas em Deep Learning, como é o caso de abordagens que usem Redes Neurais Convolucionais (CNNs), (iii) desenvolver uma metodologia que realize a segmentação simultânea de imagens de uma mesma pessoa em múltiplos protocolos, como frontal, lateral e outros ângulos.

\section{REFERÊNCIAS}

[1] W C Amalu, W B Hobbins, J F Head, and R L Elliot. The biomedical engineering handbook-medical devices ans systems. Infrared The Biomedical Engineering Handbook-Medical devices ans systems, 3, 2006.

[2] Matheus Baffa, Deivison Cheloni, and Lucas Lattari. Segmentação automática de imagens térmicas das mamas utilizando limiarização com refinamento adaptativo. In Anais Principais do XVI Workshop de Informática Médica, pages 39-48. SBC, 2016.

[3] A. Chagpar. An introduction to breast cancer. (11m03s), coursera., 2018 Disponível em: https://www.coursera.org. Acesso em: 10/03/2020.

[4] Navneet Dalal and Bill Triggs. Histograms of oriented gradients for human detection. In 2005 IEEE computer society conference on computer vision and pattern recognition (CVPR'05), volume 1, pages 886-893. IEEE, 2005.

[5] Maíra Araújo de Santana et al. Breast cancer diagnosis based on mammary thermography and extreme learning machines. Research on Biomedical Engineering, 34(AHEAD):45-53, 2018.

[6] Hospital do Câncer de Barretos. Informação: Saiba quais são os principais tipos de câncer mais comuns no brasil, 2018. Disponível em: https://www.hcancerbarretos.com.br/82-institucional/noticiasinstitucional/1300-\%20informacao-saiba-quais-sao-os-tipos-de-cancermais-comuns-no-brasil. Acesso em: 10/03/2020.

[7] Instituto Nacional do Câncer INCA. Estatísticas de câncer, 2020 Disponível em: https://www.inca.gov.br/numeros-de-cancer. Acesso em: 02/08/2020.

[8] ABC Fonseca, ESRC Rodrigues, MM Nóbrega, JOC Nobre, GJ França, and LP Silva. Estimativa para o câncer de mama feminino: e a assistência de enfermagem na prevenção. Temas em saúde, 16(4):14-30, 2016.

[9] GLOBOCAN. Cancer fact shets: Breast cancer, 2020. Disponível em: http://globocan.iarc.fr/old/FactSheets/cancers/breast-new.asp. Acesso em: 10/03/2020

[10] Jay P Gore and Lisa X Xu. Thermal imaging for biological and medical diagnostics. In Biomedical Photonics Handbook, pages 540-553. CRC press, 2014.

[11] INCA. Tipos de câncer - câncer de mama, 2020. Disponível em: https://www.inca.gov.br/tipos-de-cancer/cancer-de-mama. Acesso em: 02/08/2020.

[12] Afsaneh Jalalian, Syamsiah Mashohor, Rozi Mahmud, Babak Karasfi, M. Saripan, and Abdul Ramli. Foundation and methodologies in computer-aided diagnosis systems for breast cancer detection. EXCLI Journal, 16:113-137, 022017.

[13] J Josephine Selle, A Shenbagavalli, N Sriraam, B Venkatraman, M Jayashree, and M Menaka. Automated recognition of rois for breast thermograms of lateral view-a pilot study. Quantitative InfraRed Thermography Journal, 15(2):194-213, 2018.

[14] Siva Kakileti, Geetha Manjunath, Himanshu Madhu, and Hadonahalli Ramprakash. Advances in Breast Thermography. 102017.

[15] Sebastien Mambou, Ondrej Krejcar, Petra Maresova, Ali Selamat, and Kamil Kuca. Breast cancer detection using infrared thermal imaging and a deep learning model. Sensors, 18:2799, 082018.

[16] R. Marques. Segmentação automática das mamas em imagens térmicas, 2012.

[17] Antony Morales, E. Kolosovas, Edgar Guevara, Mireya Maruris Reducindo, Alix Hernández, Manuel García, and Francisco Gonzalez. An automated method for the evaluation of breast cancer using infrared thermography. EXCLI Journal, 17:989-998, 102018.

[18] L. Motta. Obtenção automática da região de interesse em termogramas frontais da mama para o auxílio à detecção precoce de doenças., 2010. Dissertação de Mestrado, Universidade Federal Fluminense, Instituto de Computação, Niterói, RJ, Brasil.

[19] J. Oliveira. Extração automática de região de interesse em imagens térmicas laterais da mama., 2012. Dissertação de Mestrado, Universidade Federal Fluminense, Instituto de Computação, Niterói, RJ, Brasil.

[20] Proeng. Image processing and image analyses applied to mastology., 2012. Disponível em: http://visual.ic.uff.br/en/proeng/. Acesso em: $10 / 03 / 2020$. 